\title{
Survey on UAV Communication Network: Performance Parameter
}

\author{
Vidyashree D \\ Department of Electronics and Communication \\ Engineering \\ JSS Science and Technology University \\ (Formerly SJCE) Mysuru, India
}

\author{
Kavyashree M K \\ Department of Electronics and Communication \\ Engineering \\ JSS Science and Technology University \\ (Formerly SJCE) Mysuru, India
}

\begin{abstract}
Unmanned aerial vehicles (UAVs) have enormous potential within the public and civil domains. These are particularly useful in applications, where human lives would preferably be endangered. It is utilized to produce flexible wireless access in future wireless networks, with larger coverage and better transmission rate. Most of the work carried out in the areas of mobile ad hoc networks (MANETs), and vehicular ad hoc networks (VANETs) does not address the unique characteristics of the UAV networks, while it is believed that ad hoc mesh network would be most suitable for UAV network. The Unmanned aerial vehicles (UAVs) is deployed efficiently to produce prime quality of service for Internet of Things (IoT). Protocols are required that would adapt to high mobility, dynamic topology, intermittent links, power constraints, and changing link quality. As energy supply on UAVs is limited, protocols in various layers should contribute toward greening of the network. This paper describes different work done towards all of these issues on how UAVs are utilized in identification and tracking of UAV nodes, Energy consumed by nodes and routing strategy in several network in several applications. The wireless backhaul for UAVs is sometimes capacity-limited and congested, and UAV nodes cannot operate for a protracted time because of the limited battery life. Finally, Identifying the challenges in UAV enabled network.
\end{abstract}

Keywords- Unmanned Aerial Vehicle (UAV), Internet of Things (IOT), Energy Efficiency, Trajectory paths, QOS, Routing wireless network.

\section{INTRODUCTION}

Unmanned Aerial Vehicle (UAV) is an emerging technology that can be utilized for military and civil applications. The UAV delivers the data at every time even if there is any disaster warnings and gets assisted in speeding of rescue and recovery operations when the general public communication network gets crippled. In situations like poisonous gas infiltration, wildfires and wild animal tracking UAVs can be configured by software's via remote controller, to provide medical help to areas rendered inaccessible and is easier to envelope enormous area without risking the safety of the personnel involved. UAV's are likely to become invaluable inclusions within the operations of police departments, fire brigades and other offices of homeland security organizations soon. As there is advances in electronics and sensor technology, this have paved a way to widen the scope of UAV network applications such as traffic monitoring, wind estimation, natural fire detection, and remote sensing. The swarm of UAVs will be working cooperatively to accomplish complex tasks in enormous area in search and rescue operations, which is the future application of the UAVs.

Internet of Things (IoT) is another emerging technologies and some new emerging ICT technologies such as the 5G devices/sensors convers variety of application fields. $5 \mathrm{G}$ is a new generation of mobile communications technology. Its integration with firms to communicate and control with smart machines, building by applying collaborative automation and intelligent optimization. This $5 \mathrm{G}$ technology is a unifying fabric that will connect billions of devices in faster and more reliable and most efficient ways as possible.

This paper gives a survey on the UAV network with more efficiently by considering quality of service (QoS) in terms of network lifetime, energy consumption, delay and throughput. how to improve the stability of route. It presents the UAVs issues and challenges in the cached UAV-enabled in smallcelled network.

For example, in a forest fire scenario, a swarm of UAVs equipped with different sensors need to be deployed efficiently to establish an emergency communication system, collecting different types of data (such as temperature, pressure, video, etc.). Since there is a limit in the transmit power of UAV, designing a reliable and efficient UAVs relay network to guarantee a good coverage for the monitoring data collection in a large area. The low latency demand for the disaster rescue scenario for the efficient information collection is crucial for a swarm of UAVs from various data collecting devices to the data processing center. The dynamic characteristics of UAVs network also bring new challenges to the stability of data transmission. Therefore, in order to establish an efficient UAV-assisted emergency communication system, there are many challenging problems unsolved, such as the network architecture design for an efficient cooperative communication among UAVs, and the low latency routing algorithm with dynamic network topology constraints.

\section{LITERATURE SURVEY}

[1] This paper portrays the Internet of Things (IoT) enable Unmanned Aerial Vehicles (UAV) for industrial-oriented applications for multimedia, video streaming delivery plays key role. This industrial IOT embedded sensors are placed everywhere. Connects with several things in real world through diverse communication system which is capable of monitoring, collection, and delivering valuable amount of data. Here these UAV which are involved play major role in Industrial-Internet of Things (IIOT) related application. This 
paper mainly concentration multipath routing of IIOT for recovering selecting and construction of k-disjoint paths and by considering the Quality of Service. By introducing industrial oriented Canonical Particle Swarm (CPM) optimization data framework. The fault-tolerant system in two-tiered heterogeneous Wireless Sensor Network (WSN). Where it comprises of super/smart node which has a greater number of resources and simple/high sensor nodes with limit in their battery life and Quality of Service (QOS) limitation. For a k-disjoint multipath routing approach they look at many to one traffic system. Where those two nodes connect with proper degree of isolation. For every disjoined/isolated node they have used a k-disjoint multipath route which, increases the number of marginal paths. As, it is interpreting a fault tolerant network. This model paper shows that a node can connect or disconnect with some nodes that are not among the k-disjoint multipath between the super node and common node. The model constructed in this paper gives a way that each sensor node in network in within range of transmission. This mainly helps in the minimization of QOS parameters as transmission power and latency will be maintained along with k-disjoint multipath route. The transmission range should be main as amount of energy in used as k-disjoint multipath and all parameter for QOS is upheld to resolve the fault-routing problem from convergence issue. To offer quick recovery from failure via multipath routing capability of attaining QOS by estimating energy consumption, lifetime, delay in transmission and overall through put parameters they have implemented aforementioned algorithms that is similar that of canonical particle multipath swarm optimization (CPMS) and canonical particle swarm optimization and the fully particle multi-path swarm optimization (FMPS) is used to offer the different learning technique for swarm particle, using the MATLAB to evaluate the parameters. This paper finally, conclude by finding the $\mathrm{k}$-disjoint multipath routes in network of UAVs by using the algorithms that recover and constructs then and proposed algorithm is compared with similar algorithm to optimize the energy consumption and overall delay on the path from source to destination. This paper gives a study of 5G/IOT integration with sensors in network for industrial application.

[2] This article gives complete description about the framework of caching of UAV in a small-celled base station via caching. This paper mainly concentrates on three limitations in UAV enabled networking. Firstly, Wireless backhaul this is where the UAV provide data services to mobile users with backhaul links which connects to core network. Hence users are limited by the capacity of backhauls during peak periods. Battery limitations as UAVs cannot depend on the fixed power supply UAV communication energy aware algorithm should be developed to save energy. Mobility limitations make the UAV to estimate channel state information accurate. To solve the above limitations, they have proposed method caching. Where the caches mounted at UAV will be cached during the off-peak period at first. It will be picked up and transmitted to mobile users directly from local caches. The UAVs can be reloaded with contents either through wireless backhaul from macrocell base station as in framework. The UAVs broadcasts cached segments of required contents to other small cell UAVs due to popularity this helps in small cell base station offload data traffic from local cache. As this happen in Device to Device (D2D) communication. This paper also explains the mobile caching UAVs base station without ground Small celled Base Station's (SBS). Where this helps the Base Station (BS) to have higher transmission rate and reduce the load on wireless backhaul. Caching UAV offloading for SBS's has simultaneous services and separated services by SBS and UAV. In the simultaneous services when there is less number of Small cell User (SU), in small cell network the UAV helps in SBS offload data to SU. Whereas in separated service when there are plenty of SUs in small cell with a few UAVs network gets congested and SBS cannot support the transmission data task at times. They have addressed some research issues and challenges on caching UAV-enabled small-cell network. Firstly, optimal caching strategy in important to improve the caching efficiency and guarantee the transmission performance secondly, in popularity analysis is one of the key challenges in this area to popular context for local small cell UAVs. Which determines what context of data to be stored with in the limited local caches at UAVs. Thirdly, communication security as UAV may carry sensitive information so that should not be eavesdropped at any point of time, attacks can happen by jamming, WIFI attacks, GPS spoofing. The Energy saving is another challenge in UAV communication due to its less battery capacity. As explained in this paper, caching can help UAVs at congestion of wireless network, thus by saving energy and lastly joint optimization and interference issues also may break the wireless network. Finally, the popular controls are cached at UAVs during speak period, which is fetched directs to help SBS to avoid data traffic at SUs at peak time. Thus, this paper finally explains the two techniques UAV and caching will be combined to improve the small-cell network performance. This, overload of backhaul for UAV can be revived via caching and energy consumption can be improved.

[3] This article gives a unified framework of UAV assisted emergency network in a disaster. The flight trajectory and communication scheduling of UAV is optimized to give wireless service for mobile devices with the surviving ground base stations (BSs). The multi-hop D2D and transceivers designed of UAV without ground BS's to efficiently cover the wireless coverage of UAV to realize the information exchange between disaster and outside are in these scenarios. The multi-hop UAV relaying scheme is used to optimize the hovering positions of UAV. This article is explained using scenarios. Firstly, with active ground BS's UAVs can communicate with surviving BS's to provide wireless service to ground devices. At this situation the flight trajectory and communication scheduling can be jointly optimized, to improve performance of the system. second scenario is where with no BSs a number of UAV can be used as flying BS to provide wireless communication, help of multi-hop D2D to extend coverage area. Then, multi-hop UAV relaying is scenario where the information is exchanged between disaster areas which are outside the scenarios 1 and 2 is realized via UAV multi-hop relaying. this derives optimal hovering positions of UAV to low complexity. The joint trajectory and scheduling optimization are used to reach the BS when they are partially damaged. The work on UAV's trajectory 
transmit power and scheduling without any surviving ground BS's. due to the better mobility the UAVs can navigate closer to ground devices to achieve better performance which causes interferences to devices. In complex situation the interferences between BS-served devices and UAV -served devices should be avoided for reliable transmission. To achieve guaranteed reliable transmission and avoid interference to the BS-served devices. UAV's fly periodically at a fixed altitude with flying cycles multiple antennas are placed at each BS, then the UAV's are equipped with single antennas. The classic optimization algorithm is used to solve the optimization of surviving BS's to UAV, which is extremely difficult solve due to the mixed integer non-convex problem. Hence this algorithm is used. As there is a limited power transmit of UAV the QOS of edge devices is difficult. Hence multiple antennas at UAV should be fully exploited for reliable transmission. As to design and establish multihop D2D, the numbers of hops should be minimized with reliable performance. Each hop can store power due to power limitations. To resolve thus shortest-path routing (SPR) algorithm in designed as it can select a device as relay node if it is near to destination than other devices, with coverage of radius of current node to guarantee reliability. This SPR algorithm is suitable for establishing multi-hop D2D links to extend coverage UAV with less hop cycles. The challenges addressed are multi-UAV trajectory, interference management, channel modeling, energy supply. Finally, this paper gives overall information about how the exchange of information between disaster area outside world its optimized hovering positions of UAVs as a multi-hop relaying schema and UAV assisted emergency issues challenges.

[4] IOT enabled UAV, this paper gives the network architecture and routing algorithm the layered UAV swarm network architecture is proposed and analyzed with minimum number of UAVs gives the partial information of location and network connectivity of UAVs. The LLRA algorithm is designed for this layered architecture in the proposed system. This LLRA algorithm distributes different data traffic flows dynamically via optimal relay which, minimize the latency efficiently. The layered network in this paper gives a system model which solves the low latency routing problem formation in UAV enabled IOT. To minimize the transmission latency problem in large swarm of UAVs. This model is designed. This pages also solves the low latency routing optimization scheme by considering the location and connectivity of UAV's. To address these problems the UAV deployed with high QOS for IOT. The system model can mainly be used in situation like earthquake or forest fire. Where the UAV-base communication network will be used to collect information from IOT sensor devices and transmit it in real time via video-streaming and also sends to ground level fire fights. As UAV network topology have challenges to UAV network performance and effective routing optimization among UAV's. In this model different types of UAV is placed by considering diversity of coverage and flying capability speed among UAV at different levels upper layer has fixed UAV circulating which provide effective coverage to UAVs at lower layer by directional antennas upper layer act as relay node to assist the communication of lower layer and reduce delay during transmission lower layer consists of different IOT sensors which are helpful in collecting and transmitting the sensed data in a disaster area. To detect the occurrence of probability and possibility of forest fire and predict speed fire spread timely, how efficient the data in traversed between lower to upper layered nodes. This arise a low latency communication between lower layer UAV's is crucial therefore, LLRA algorithm is designed based on location and connectivity information of UAV in order to improve routing performance. Finally, this paper evaluates the performance of LLRA routing algorithm in layered architecture and the key parameter also analyzed. This algorithm gives the link average delay and packet delivery ratio. The results prove that the LLRA algorithm gives better performance than traditional AODV and GPRS algorithm.

[5] This paper proposes a layered directional networking and rate-distortion power optimized efficiently to transmit the encoded data and overcome network transisnts which leads to packet buffering. In this paper the UAVs are linked to a ground-based station over direct network links of shared aggregated data rate. The link capture data towards aggregation point and controls information which passes through drones. This system has immersion fidelity to deliver the user which depends on space time position of UAV-IOT network. The data that captures or transmits to the user navigation action. Maximizing application constraints raises multiple technical challenges due to technology limitations to capture coding networking and reconstruction of VR data . This proposed system framework has four integrated components which depends scope towards next generation application. The VR immersion fidelity formulates the user view port-driven remote scene for UAV IOT to capture data for spatial UAV positions by optimization problem of VR application latency/internality constraints UAV-IOT network placement to maximize the expected VR immersion fidelity delivered over time. The sealable joint source-channel viewpoint coding maximizes the reconstruction fedility of data captured by each UAV. Thus, layered directional power is optimized to embedded scheduling for effective transmission of encoded data. Thus, efficient online learning decides the optimal dynamic placement of UAV-IOT network. This maximizes the immersion fedility to deliver to VR user. The learning interacts closely with other system components. This selects the minimum UAV data capture rate over time by spatial location. Thus, acquired data in efficiently represented using source-channel viewpoint coding to maximum reconstruction aggregate point. The data is encoded effectively transmitted using layered directional network and rate distortion-power optimized transmission scheduling. The online learning optimization is carried out at the aggregation point server computer attached. Finally, this paper explored layered directional networking and ratedistortion-power is optimized. They have explored efficient online leaning in order to discover better dynamic UAV-IOT network placement and to maximize the delivered expected immersion fedility. They have demonstrated the network VR performance efficiency which gains enabled system component over state-of-the-art method delivered VR immersion fedility and transmission power consumption. 
[6] This paper defines the maximization energy efficiency for a single UAV carrying wireless power transfer. The contribution of this paper summarized, for this they have proposed a concept of UAV energy efficiency utilization in order to analyze the UAVs trajectory optimization problem. Two practical charging strategies between UAV and Sensor Node (SN), where the UAV charging point is fixed, and other situation is that power transfer efficiency is unchangeable within a certain distance. Then decomposing the maximum UAV energy utilization problem into integer programming problem and non-convex-optimization problem. So, previously they had presented minimum 1-tree to limit search direction to provide better initial search position also adopt local search method to reduce the search space. They have used Trajectory Planning Algorithm with Fixed Charging Position (TPA-FCP) to solve the problem of UAV charging position is fixed and to speed up the performance of algorithm by limiting the search direction and search space. At situation where the power transfer efficiency is unchangeable within a certain distance. They introduced Polynomial-time Randomized Approximation Scheme (PRAS), to discovery the minimum number of hovering locations. This paper also shows that the TRA-ERC has best performance in finding the shortest trajectory and TPA-FCP is algorithm which takes the least amount of total time in optimal trajectory computation. Finally, the result shows the improvement in the UAV energy utilization efficiency to specify charging trajectory optimization problem in a largescale network.

[7] This paper explains role of UAV in public safety communication. It starts with a multi-layered architecture will be used in public safety communication. where, the UAV plays an important role in providing conventional communication services at any disaster situations. In those situations when there is no possibility of communications with no alternative path is available due to damage in Terrestrial Base Station (TBS). Hence the authors have adopted energy- efficient uplink communication for UAV assisted mobile architecture to collect data from IOT devices. This paper will not give any solution to use but it explains the issues involved in the optional trajectories and low latency and routing challenges in various UAV network. To overcome the problem, they have proposed multilayered architecture which is capable of providing alternate path if any path fails, several cables are also deployed to enhance the signal quality in the ground and underground layers. To overcome wireless communication failure in basement separate cable in routed this is a special feature of this architecture. These finally in air-layer UAVs connected to each other also connected to ground layer uses and base station to coverage the disaster area even when TBS is destroyed completely. This also increases the energy efficiency by decreasing latency, UAV also used to enable edge/fog computing. Then this paper explains the important factors affecting UAV's energy consumption some factors explained here are energy efficiency UAV placement. UAVuser association in emergency communication. UAV scheduling optimization. Also addressed the issues and challenges for future research that are firstly UAV flying path planning challenges. This path planning has many problems like collision avoidance, energy consumption, trajectory cost The intelligent optimization algorithm is used to resolve this issue. Then secondly, privacy, security and safety challenges. From safety point of view, it is important to design mechanism for safe and targeted landing of device in emergency situation. The artificial intelligence technique provide solution to address security in UAV connected IOT applications. Thirdly, UAV recharging automation this problem can be resolved by using the most recent energy harvesting techniques using solar energy, but it has less efficiency to enhance the performance of energy efficiency distribute multipoint wireless power technique is most useful Finally, this paper concludes by proposing the multilayered architecture to enable public safety communication that has capacity of enabling communication in basement which helps in both wired and wireless communication. They have also addressed factors affecting UAV in energy consumption and the major issues and challenges in research direction of UAV.

[8] This paper portrays the UAV enabled mobile edge computing (MEC) system for powering the UAV nodes. The Wireless Power Transfer Technology (WPT) utilized by UAV to first power the IOT Device. Then each of Internet of Things Devices (IOTD) sends and collect data to UAV for processing by energy harvested from UAV. In some of the IOTD there will be limited or no computation capability. Because of their limited sizes to process its collected data and respond to environmental changes is difficult because the IOTD is may be too far from energy sources and difficult to change conveniently and cost effectively. As the MEC and WPT provides solution to the problem in this paper. This paper they have considered as, the IOTD's are powered by using the moving energy sources of UAV and this UAV are installed with single antenna and central computing unit. These UAV's files above the IOTD from one place to another back to initial location. However, In the m UAV assisted system that contains one workflow to complete tasks in conventional working manner which is inefficient compared to multiple system. Hence, in this paper a new TDMA base workflow for UAV is used to minimize UAV energy consumption, IOTDs association, computing resource allocation, wireless powering duration uplink data rate and service sequence which allows parallel transmission and execution in UAV system. In order to extend the serving time of UAV and to enhance the energy efficiency. TDMA workflow mode is developed it also has multiple workflows and allows parallel operation. The UAV energy minimization problem in proposed model is resolve by using Block Coordinate Descent Method (BCD). They have used flow-shop scheduling technique the resolve the flow shop problem by obtaining optimal IOTDs sequences. Finally, this paper gives an overview of how they have formulated UAV minimization problem as mixed-integer non-convex problem, which is solved by transforming and relaxing it by using flow- shop scheduling technique. Then the alternative algorithm is introduced to set initial points close to optimal results. This paper's result shows that enhancement in the energy compared to conventional UAV assisted system. 
[9] In the paper, they have proposed a framework for deployment and mobility of multiple efficiently deploying and moving UAVs nodes in order to collect data at uplink from ground IoT devices. They have determined optimal UAV locations, device associations and uplink power control over IOT devices. The total transmit power of devices is minimized under the Signal-To-Interference-Plus-NoiseRatio (SINR) constraints. This proposed framework consists of two steps. Firstly, at any locations of the IOT devices. They have proposed a solution for optimizing and deploying the UAV there in this case to solve the formulated problems by decomposing it into two subproblems. These subproblems divided to solve iteratively. First subproblem, give constant UAVs location, to find jointly optimal device UAV association and device transmit power. second subproblem, gives fixed device association to determine the joint 3D UAVs location. For these subproblems they have transform the non-convex location optimization problem to convex form provide traceable solutions. This proposed approach leads to an efficient solution with reasonable accuracy to global optimal solution overhead. The second step is to analyze the IOT network over a period of time where an active device change. The framework optimizes the UAVs mobility by allowing to timely vary mobility by allowing to timely vary devices for activation process. The UAV deployment and device association with power control is also considering here. The UAV's location must be known in order to find device associations. The UAV location cannot be optimized without device association they have decomposed two sub problems that will be solved iteratively. First subproblem, then the second sub problem. They determined sub optimal location of UAV where the transmit power is minimizing. This is an iterative process where the results of each sub problem used in next subproblem iteration. These computations are performed by 3D UAV location by control until. The limited number of orthogonal Channels which acts as interferences between the device will mainly depend on the number of active devices at each time. When there is no interferences between active devices at time then there is less number of orthogonal channels. This paper has investigated the interference and interference free scenario for device association power control, optimal location of UAVs. Then they found the optimal update time and trajectory of UAV reliable uplink transmission IOT device. These trajectories of UAV and their update time depends on activation process of IOT devices. The move the UAV must spend minimum total energy on mobility. So, as to remain working for longer time. They have investigated the effective motion of UAV to collect information in time varying IOT network based on devices activation process the Update tine at each instance are used by UAV to dynamically serve different IOT devices with min energy consumption. Finally, this depicts the total transmit power of IOT device by $45 \%$.

[10] This paper proposed in order to improve to QOS requirements. They have considered a three-tire hybrid network where UAV cooperates with satellites and base station (BS) which gives a space-air-ground-three-tier heterogeneous network. This is beneficial for providing seamless coverage and also improves the capacity of IOT network. This paper they worked on resource allocation for UAV network in context of these three tiers by considering feasible deployment of drones in order to reduce the cross their interference. The second stage joint hovering altitude and power control solution have been proposed in this model. Where it uses both the lagrange dual decomposition and concave-convex procedure (CCP) method used to relaxed convex problem. This uses the low-complexity greedy proportional power constrained algorithm for resource allocation in network. The algorithm implemented in this paper for UAV hovering altitude aided resource allocation. To even reduce the computational complexity, they have used heuristic resource allocation algorithm. The simulation of this proposal system evaluates the performance of two stage joint resource allocation scheme and shows how it is beneficial in terms of improving UAV network total through put by crosstier interference. This paper yield with low complexity proportionable power constrained resource allocation and performance of UAV networks through put.

[11] This paper, proposes a practical fly-hover-andcommunication-protocol for UAV enable multiuser communication the UAV play a significant role in wireless system in the applications like data offloading for Cellular Base Stations (CBS) in hotspot areas. As in the existing work of UAVs are equipped with either omni directional antenna or direction antenna with fixed bandwidth. In this article they have considered a new scenario, where UAVs are equipped with directional antenna whose bandwidth can be adjustable. For UAVs with tunable antenna bandwidth, inserting tradeoff in adjusting the antenna bandwidth of UAV v/s the altitude of it ground position. The UAVs altitude increasing the antenna bandwidth helps to cover a greater number of ground terminals (GT) by UAVs within antenna lobe. At the cost of reduced line capacity for each GT. When there is an increasing in the UAV bandwidth which covers more GTs but with lower link capacity for each GT due to increase link distance. In order to resolve tradeoff, they have proposed the fly-hover-and-communication protocol. Here the GTs are divided into disjoint clusters. Each cluster determines area projected by antenna main lobe on the ground-each and every cluster is served by UAV as it hovers above the cluster center. They have studied three fundamental UAV multiuser models, that are downlink multicasting(MC) in this the UAV sends data which is common to all GTs in each cluster downlink broadcasting(BC), UAV sends independent information to different GTs via Frequency Division Multiple Access(FDMA), finally uplink multiple access(MAC), each GTs sends independent information to UAV via FDMA. Finally, result shows that optimal UAV altitude and antenna beamwidth. Interms of UAV altitude, it should be set to maximum possible value for downlink, MC minimum values for downlink $\mathrm{BC}$ whereas it can have feasible values for MAC. Antenna beam width should be set to minimum feasible value for downlink MAC. This papers result, drastically shows the setting optimal altitude and beamwidth values in different multiuser models.

[12] This paper gives an insight view of energy efficiency for NOMA UAV communication system by accounting both transmission power and energy consumption during flying operation. the power domain Non-Orthogonal Multiple 
Access (NOMA) scheme for energy efficiency of UAV BS's. This paper evaluates the effect of paired users on operational altitude of UAV-BS. By using this pairing between user and UAV BS with best and work channel condition. They have determined a methodology for feasible altitude range of UAV-BS for each user rate is satisfied with prescribed transmission power limit. The low complexity method to determine the constrained altitude bounds for the dependency of the feasible range on subset of user pairs is proposed in this paper. They solved by employing an alternative optimization technique. They solved maximization problem by fixing the altitude of power allocation to feasible value. Then power allocation to feasible valve. Then power allocation is optimized to maximum by fixing altitude of CAV-BS. To solve NLFP and to provide guarantee convergence within an error tolerance limit. The power allocation of proposed NOMA based UAV communication system are evaluated at close optimum altitude. The OMA scheme gives better performance in proposed methodology. This paper investigate NLFP of energy efficiency max as function NOMA performs better than OMA is defined in this paper. The methodology in this paper define a range of UAVBS altitude by ensuring the rate of constraints is satisfied by transmission power. This proposed system gives up to $18 \%$ power saving by taking signal transmission in to account with minimum power saving of up to $49 \%$. Then the energy efficiency improves more that $65 \%$ and 100 in sub-urban and dense-urban deployment.

[13] In this paper, they proposed a computation rate maximization in UAV-enabled MEC wireless. The formulate framework for resource allocation in UAV-enabled MEC wireless powered. System uses both partial and binary computation of offloading nodes. The computation bits are maximized by optimizing the CPU frequencies, the transmit power and offload time also uses the UAV trajectory path. The two-stage alternative algorithm is proposed in this paper to solve the maximization problem at non-convex and challenging computation bits. The problem of maximization weighted sum computation bits for the three-stage alternative algorithm is proposed. The offload tasks of UAV for computation depend on tradeoff between the computation rate and operation cost. The trajectory of UAV is optimized successive convex approximation method under the binary and partial computation offloading modes. By using resource allocation scheme is better than the disjoint optimization scheme to achieve better computational performance. It takes several iterations for proposed algorithm to coverage. This paper concludes by providing simulation results to verify the priority and fairness of user is improved by using weight vector. The closed-form expressions for the optimal central processing unit frequencies, user offloading time, and user transmit power are derived in this paper.

The below is a comparison table that includes overall summary of the survey of the research.
TABLE I. COMPARISION TABLE

\begin{tabular}{|c|c|c|c|}
\hline Year & Title & Approach & Description \\
\hline 2018 & $\begin{array}{l}\text { 5G/IoT-enabled } \\
\text { UAVs for } \\
\text { multimedia } \\
\text { delivery in } \\
\text { industry-oriented } \\
\text { applications }\end{array}$ & $\begin{array}{l}\text { Aforementioned } \\
\text { Algorithm have } \\
\text { different learning } \\
\text { contrivances and } \\
\text { increases the number of } \\
\text { paths that is required to } \\
\text { exchange the message. } \\
\text { To evaluate the } \\
\text { parameter, they have } \\
\text { used MATLAB. }\end{array}$ & $\begin{array}{l}\text { The proposed } \\
\text { algorithm optimizes } \\
\text { the energy } \\
\text { consumption and } \\
\text { average delay on } \\
\text { the explored paths } \\
\text { towards the } \\
\text { destination. }\end{array}$ \\
\hline 2018 & $\begin{array}{l}\text { Caching UAV- } \\
\text { Enabled Small- } \\
\text { Cell Networks }\end{array}$ & $\begin{array}{l}\quad \text { Presented a } \\
\text { framework for caching } \\
\text { UAV-enabled small-cell } \\
\text { networks. The } \\
\text { distributed caching } \\
\text { strategy for UAVs was } \\
\text { also presented. }\end{array}$ & $\begin{array}{l}\quad \text { In this paper, } \\
\text { UAV and caching, } \\
\text { have been } \\
\text { combined to } \\
\text { improve the } \\
\text { performance of } \\
\text { small-cell } \\
\text { networks. A case } \\
\text { study } \\
\text { Interference also } \\
\text { presented. }\end{array}$ \\
\hline 2019 & $\begin{array}{l}\text { UAV-Assisted } \\
\text { Emergency in } \\
\text { Networks } \\
\text { Disasters }\end{array}$ & $\begin{array}{l}\text { To improve the } \\
\text { transmission efficiency } \\
\text { of UAV classic } \\
\text { optimization } \\
\text { Algorithms and Shortest } \\
\text { Path Routing (SPR) to } \\
\text { establish best path. }\end{array}$ & $\begin{array}{l}\text { This paper gives a } \\
\text { complete review of } \\
\text { multi-hop relaying } \\
\text { scheme in order to } \\
\text { exchange the data } \\
\text { between the area or } \\
\text { disaster and } \\
\text { outside. Where, } \\
\text { hovering positions } \\
\text { of UAV } \\
\text { optimized. }\end{array}$ \\
\hline 2019 & $\begin{array}{l}\text { IoT Enabled } \\
\text { UAV: Network } \\
\text { Architecture and } \\
\text { Routing } \\
\text { Algorithm }\end{array}$ & $\begin{array}{l}\text { Low Latency Routing } \\
\text { Algorithm (LLRA) is } \\
\text { used to achieve the } \\
\text { optimal route with the } \\
\text { minimum delay and } \\
\text { dynamically distribute } \\
\text { data traffic flows. }\end{array}$ & $\begin{array}{l}\text { Layered UAV } \\
\text { swarm network } \\
\text { architecture } \\
\text { proposed, and an } \\
\text { optimal number of } \\
\text { UAVs } \\
\text { analyzed. } \\
\text { Performance of this } \\
\text { LLRA is verified to } \\
\text { improve the packet } \\
\text { delivery ratio. }\end{array}$ \\
\hline 2019 & $\begin{array}{l}\text { UAV-IoT for } \\
\text { Next Generation } \\
\text { Virtual Reality }\end{array}$ & $\begin{array}{l}\text { Fast Explore and } \\
\text { Exploit Learning } \\
\text { (FEEL) Algorithm is } \\
\text { implemented to VR } \\
\text { immersion. }\end{array}$ & $\begin{array}{l}\text { This paper } \\
\text { demonstrates the } \\
\text { networked } \\
\text { performance } \\
\text { efficiency and in } \\
\text { delivered VR } \\
\text { immersion fidelity, } \\
\text { application } \\
\text { interactivity/play- } \\
\text { out latency, and } \\
\text { transmission power } \\
\text { consumption. }\end{array}$ \\
\hline 2020 & $\begin{array}{l}\text { Trajectory } \\
\text { Optimization for } \\
\text { UAVs' Efficient } \\
\text { Charging in } \\
\text { Wireless } \\
\text { Rechargeable } \\
\text { Sensor Networks }\end{array}$ & $\begin{array}{l}\text { TPA-FCP Algorithm } \\
\text { and Polynomial-time } \\
\text { Randomized } \\
\text { Approximation Scheme } \\
\text { is used to solve the total } \\
\text { time in optimal } \\
\text { trajectory computation. }\end{array}$ & $\begin{array}{l}\text { The proposed } \\
\text { shows the } \\
\text { improvement in the } \\
\text { UAV energy } \\
\text { utilization } \\
\text { efficiency to } \\
\text { specify charging } \\
\text { trajectory } \\
\text { optimization } \\
\text { problem in a large- } \\
\text { scale network. }\end{array}$ \\
\hline 2019 & $\begin{array}{l}\text { Role of UAVs in } \\
\text { Public Safety } \\
\text { Communications: } \\
\text { Energy } \\
\text { Efficiency } \\
\text { Perspective } \\
\end{array}$ & $\begin{array}{l}\text { Multi-Layered } \\
\text { Architecture to enable } \\
\text { energy efficient uplink } \\
\text { communication. }\end{array}$ & $\begin{array}{l}\text { Proposed the multi- } \\
\text { layered architecture } \\
\text { to enable public } \\
\text { safety } \\
\text { communications, } \\
\text { which has the }\end{array}$ \\
\hline
\end{tabular}




\begin{tabular}{|c|c|c|c|}
\hline & & & $\begin{array}{l}\text { capacity to enable } \\
\text { communication in } \\
\text { basement using } \\
\text { wired or wireless } \\
\text { communication. }\end{array}$ \\
\hline 2019 & $\begin{array}{l}\text { Joint Resources } \\
\text { and Workflow } \\
\text { Scheduling in } \\
\text { UAV-Enabled } \\
\text { Wirelessly } \\
\text { Powered MEC } \\
\text { for IoT Systems }\end{array}$ & $\begin{array}{l}\text { Time Division Multiple } \\
\text { Access (TDMA) is used } \\
\text { to allow parallel } \\
\text { transmissions and } \\
\text { executions in the UAV- } \\
\text { assisted system. }\end{array}$ & $\begin{array}{l}\text { Proposed } r \text { system } \\
\text { aims to minimize } \\
\text { the total energy } \\
\text { consumption of } \\
\text { UAV. }\end{array}$ \\
\hline 2017 & $\begin{array}{l}\text { Mobile } \\
\text { Unmanned } \\
\text { Aerial Vehicles } \\
\text { (UAVs) for } \\
\text { Energy-Efficient } \\
\text { Internet of } \\
\text { Things } \\
\text { Communications }\end{array}$ & $\begin{array}{l}\text { Iterative algorithm is } \\
\text { used in order to solve } \\
\text { the subproblems }\end{array}$ & $\begin{array}{l}\text { The total transmit } \\
\text { power of devices is } \\
\text { minimized under } \\
\text { the Signal-To- } \\
\text { Interference-Plus- } \\
\text { Noise-Ratio. The } \\
\text { total transmit } \\
\text { power of the IoT } \\
\text { devices is reduced } \\
\text { by } 45 \% \text {. }\end{array}$ \\
\hline 2019 & \begin{tabular}{lr} 
Joint & UAV \\
Hovering & \\
Altitude & and \\
Power & Control \\
for Space-Air- \\
Ground \\
\multicolumn{2}{l}{ Networks }
\end{tabular} & $\begin{array}{lr}\text { Low-complexity greedy } \\
\text { search algorithm and } \\
\text { Lagrange } & \text { dual } \\
\text { decomposition } & \text { and } \\
\text { concave } & \text { convex } \\
\text { procedure } & \text { (CCP) } \\
\text { method is used } & \\
\end{array}$ & $\begin{array}{l}\text { The performance of } \\
\text { resource allocation } \\
\text { yields an } \\
\text { improvement in the } \\
\text { UAV network's } \\
\text { throughput. }\end{array}$ \\
\hline 2018 & $\begin{array}{l}\text { Joint Altitude } \\
\text { and Beamwidth } \\
\text { Optimization for } \\
\text { UAV-Enabled } \\
\text { Multiuser } \\
\text { Communications }\end{array}$ & $\begin{array}{l}\text { Fly-hover-and- } \\
\text { communication- } \\
\text { Protocol is introduced to } \\
\text { resolve the trade-off } \\
\text { issue in current system. }\end{array}$ & $\begin{array}{l}\text { Different rules } \\
\text { for setting optimal } \\
\text { altitude r and } \\
\text { beamwidth values } \\
\text { in different } \\
\text { multiuser models } \\
\text { and it provides } \\
\text { useful insight for } \\
\text { UAV } \\
\text { communication. }\end{array}$ \\
\hline 2019 & $\begin{array}{l}\text { Energy-Efficient } \\
\text { Non-Orthogonal } \\
\text { Multiple Access } \\
\text { for UAV } \\
\text { Communication } \\
\text { System }\end{array}$ & $\begin{array}{l}\text { Non-Orthogonal } \\
\text { Multiple Access } \\
\text { (NOMA) and pairing } \\
\text { schema. This paper also } \\
\text { has feasibility analysis } \\
\text { and algorithm for } \\
\text { energy-efficient altitude } \\
\text { optimization and power } \\
\text { allocation }\end{array}$ & $\begin{array}{l}\text { The proposed } \\
\text { scheme achieves up } \\
\text { to } 18 \% \text { power } \\
\text { saving on signal } \\
\text { transmission with } \\
\text { maximum power } \\
\text { saving. }\end{array}$ \\
\hline 2019 & $\begin{array}{l}\text { Computation } \\
\text { Rate } \\
\text { Maximization in } \\
\text { UAV-Enabled } \\
\text { Wireless } \\
\text { Powered Mobile- } \\
\text { Edge Computing } \\
\text { Systems }\end{array}$ & $\begin{array}{l}\text { The two-stage algorithm } \\
\text { and a three-stage } \\
\text { alternative algorithm are } \\
\text { used in this paper to } \\
\text { solve the problem at } \\
\text { partial and binary } \\
\text { computation offloading } \\
\text { modes }\end{array}$ & $\begin{array}{l}\text { This proposed } \\
\text { system } \\
\text { resource allocation } \\
\text { schemes } \\
\text { outperforms and } \\
\text { the schemes } \\
\text { converge fast and } \\
\text { have } \\
\text { computational low } \\
\text { complexity. }\end{array}$ \\
\hline
\end{tabular}

\section{CONCLUSION}

UAVs have notable applications in every field of wireless sensor communication. This paper gives a brief summarization on how the role of UAV in the field of public safety communication and fire detection from energy efficiency perspective. As in some reference papers as they placed UAVs in different layers and verified according to this by analyzing the following parameters of QOS and by using unique algorithms and routing systems. Some paper explains the efficient deployment of moving UAVs to collect data with varying time in uplink of IOT network from ground level IOT devices. In such kind of situations, the optimal trajectories will be used by the IOT devices with minimum energy consumption. The design of novel scalable joint sourcechannel viewpoint coding to maximize the renovation fidelity of the UAV-IOT data captured by VR immersion at every location the UAV traverses, at the ground-based aggregation level. Finally, the VR is efferently able to provide better performance. UAV minimization problem as a mixed-integer non-convex problem so it is transformed to a tractable one that can be solved by using the convex optimization and the flowshop scheduling techniques. Then finally the development of an alternative algorithm which initial point will be set closer to the optimal solution adaptively. The simulation results of the survey paper give the energy efficiency is enhanced greatly compared with the other UAV-assisted system. Finally, about the open research issues and challenges in the UAV-assisted emergency networks have been deliberated.

\section{ACKNOWLEDGMENT}

I extremely indebted for Dr. Shankaraiah, Head of the Department of Electronics and Communication Engineering, SJCE, Mysuru, for providing me timely suggestions, encouragement and support. I'm obliged for the support presented by Asst. Prof. Kavyashree M K, Department of Electronics and communication, JSS Science and Technology University (Formerly SJCE), Mysuru.

\section{REFERENCES}

[1] Fadi Al-Turjman, Sinem Alturjman, "5G/IoT-enabled UAVs for multimedia delivery in industry-oriented applications," Springer Science, 2018.

[2] Nan Zhao, F. Richard Yu, Lisheng Fan, Yunfei Chen, Jie Tang, Arumugam Nallanathan, "Caching UAV-Enabled Small-Cell Networks", 2018.

[3] Nan Zhao, Weidang Lu, Min Sheng, Yunfei Chen, Jie Tang, F. Richard $\mathrm{Yu}$, Kai-Kit Wong, "UAV-Assisted Emergency Networks in Disasters”, IEEE Wireless Communications, volume 26, issue 1, 2019.

[4] Qixun Zhang, Menglei Jiang, Zhiyong Feng, Wei Li, Wei Zhang, Miao Pan, "IoT Enabled UAV: Network Architecture and Routing Algorithm" IEEE Internet of Things Journal, volume 6, issue 2, 2019

[5] Jacob Chakareski, "UAV-IoT for Next Generation Virtual Reality", IEEE Transactions on Image Processing, volume 28, issue 12, 2019.

[6] Pengfei Wu, Fu Xiao, Chao Sha, Haiping Huang, Lijuan Sun "Trajectory Optimization for UAVs' Efficient Charging in Wireless Rechargeable Sensor Networks", IEEE Transactions on Vehicular Technology, volume 69, issue 4, 2020

[7] Shanza Shakoor, Zeeshan Kaleem, Muhammad Iram Baig, Omer Chughtai, Trung Q. Duong, Long D. Nguyen, "Role of UAVs in Public Safety Communications: Energy Efficiency Perspective", IEEE Special Section on Mission Critical Sensors and Sensor Networks, volume 7, 2019.

[8] Yao Du, Kun Yang, Kezhi Wang, Guopeng Zhang, Yizhe Zhao, Dongwei Chen, "Joint Resources and Workflow Scheduling in UAVEnabled Wirelessly-Powered MEC for IoT Systems", volume 68, issue 10, 2019. 
[9] Mohammad Mozaffari, Walid Saad, Mehdi Bennis, Merouane Debbah, "Mobile Unmanned Aerial Vehicles (UAVs) for Energy-Efficient Internet of Things Communications", IEEE Transactions on Wireless Communications, volume 16, issue 11, 2017.

[10] Jingjing Wang, Chunxiao Jiang, Zhongxiang Wei, Cunhua Pan, Haijun Zhang, Yong Ren, "Joint UAV Hovering Altitude and Power Control for Space-Air-Ground IoT Networks", IEEE Internet of Things Journal, volume 6, issue 2, 2019.

[11] Haiyun He, Shuowen Zhang, Yong Zeng, Rui Zhang, "Joint Altitude and Beamwidth Optimization for UAV-Enabled Multiuser Communications", IEEE communication, volume 22, issue 2, 2018.

[12] Muhammad Farhan Sohail, Chee Yen Leow, Seung Hwan Won, "Energy-Efficient Non-Orthogonal Multiple Access for UAV Communication System", IEEE Transactions On Vehicular Technology, volume 68, issue 11, 2019.

[13] Fuhui Zhou, Yongpeng Wu, Rose Qingyang Hu, Yi Qian, "Computation Rate Maximization in UAV-Enabled Wireless Powered Mobile-Edge Computing Systems", IEEE Journal on Selected Areas in Communications, volume 36, issue 9, 2018. 